\title{
Reducibility Among Equilibrium Problems
}

\author{
Paul W. Goldberg* Christos H. Papadimitriou ${ }^{\dagger}$
}

August 17, 2005

\begin{abstract}
We address the fundamental question of whether the Nash equilibria of a game can be computed in polynomial time. We describe certain efficient reductions between this problem for normal form games with a fixed number of players and graphical games with fixed degree. Our main result is that the problem of solving a game for any constant number of players, is reducible to solving a 4-player game.
\end{abstract}

\section{Introduction}

Whether a Nash equilibrium of a game can be found in polynomial time is one of the most important open problems facing our field today. It is known that the problem is in $\mathrm{P}$ in the 2-player, zero-sum case, by a reduction to linear programming (12), but the general case is wide open. Very little is known about reductions between variants of the problem. It is known that finding a symmetric Nash equilibrium in a symmetric game is no easier than the general, asymmetric problem, and it is trivial, of course, to reduce $r$-player games to $r+1$-player games by introducing a dummy player (actually, to $r+1$-player zero-sum games by having the dummy player absorb everybody's payoffs). Also, evidence from Nash's original 1951 paper suggests that it may be harder to solve a 3 -player game than a 2-player game, since in the 3-player case, the resulting probabilities may be irrational. In other words, all we know about the complexity of the Nash equilibrium problem is that it comprises a potentially infinite hierarchy $2-\mathrm{NASH}, 3-\mathrm{NASH}, \ldots, r$-NASH, $\ldots$ In this paper we show that this hierarchy collapses to the 4 th level.

Games with many players require exponential data for their description; hence multiplayer games are typically represented succinctly. A most useful and influential succinct representation are the graphical games of (8), in which players are nodes of a graph, and the payoffs of each player depend only on the choices of the adjacent players. (In this paper we suggest a useful generalization, also proposed by Schoenebeck and Vadhan (15), in which the graph is directed, and player $u$ 's choice can affect the payoff of player $v$ only if edge $(u, v)$ is present.) When the degree of the underlying graph is bounded, the representation of the game is polynomial in the number of players and strategies. It has been claimed (8) that Nash equilibria in tree-structured graphical games with a fixed number of strategies per player can be computed in polynomial time. In this paper we reduce the Nash problem for graphical games to the standard version. The reduction is a polynomial one for graphical games with bounded degree (which is the intended use of graphical

${ }^{*}$ Department of Computer Science, University of Warwick, Coventry CV4 7AL, U.K. Research supported by EPSRC grant GR/T07343/01 "Algorithmics of Network-sharing Games". This work was begun while the author was visiting UC Berkeley. email: pwg@dcs.warwick.ac.uk

${ }^{\dagger}$ University of California at Berkeley, Computer Science Division, Soda Hall, Berkeley, CA 94720, USA. Research supported by NSF ITR grant and a grant from Microsoft Research. email: christos@cs.berkeley.edu 
games). More precisely, for fixed $d>1$ let $d$-GRAPHICAL NASH be the problem of finding Nash equilibria in graphical games with maximum degree $d$. We present a reduction from $d$-GRAPHICAL NASH to $d^{2}$-NASH.

We also give a reduction in the opposite direction: For any normal form game we can construct a graphical game with all degrees bounded by three and with two strategies per player such that we can recover a Nash equilibrium of the original game from any Nash equilibrium of the graphical game. Notice that the degree 3 and two strategies restriction is significant here, since otherwise the reduction is immediate (via a graphical game played on an $r$-clique).

In fact, our reduction from $r$-player games to 4-player games is a composition of these two reductions: First we reduce the $r$-player game to a graphical game, then we reduce the graphical game to a normal form game, and we obtain the 4-player result by specializing the latter reduction to the output of the former.

Our reduction from graphical games to normal form games is based on a rather simple idea: We color the graph, and simulate all vertices in a color class by a single player. This player represents the whole class by playing a mixed strategy that is the average of the mixed strategies played by the vertices in the class. In order to make sure that a color class player does not "neglect" any node by failing to include its strategies in its mixed strategy, we pair up the players, and have the pairs play against each other a generalization of Matching Pennies (see Definition 1) at very high-stakes: at any Nash equilibrium the color class player is now forced to assign the same probability mass to each vertex that it represents.

Our reduction from normal form games to graphical games is more sophisticated. Every vertex in the graphical game has two strategies, and thus, at Nash equilibrium, it can be considered as a real number: the probability that it plays strategy 1. For every player $p$ and every strategy $j$ or $p$ we have such a vertex. The challenge now is to make sure that, at any Nash equilibrium of the graphical game (a) these numbers add up to 1 for every $p$ and, more importantly (b) they encode a Nash equilibrium of the original game. (b) is accomplished by "gadgets" performing arithmetic operations such as addition (with ceiling 1, of course), multiplication, comparison, and copying. Using these gadgets we create arithmetic circuits which compute the multinomials describing the Nash equilibrium conditions of the game. Finally, (a) above is guaranteed by a hierarchy of players connected by addition gadgets.

\subsection{Review of Related Work}

It is known from $(7 ; 2)$ that it is NP-complete to find various specific kinds of Nash equilibria, even in the 2-player case. For example, it is NP-hard to find the Nash equilibrium with maximal social welfare. Kearns et al. $(8 ; 10)$ give algorithms for solving graphical games in the case where the graph is a tree and all players have 2 strategies. As for the Nash equilibrium problem itself, it is known to lie in the complexity class PPAD of (14).

Pure Nash equilibria of normal form games are of course easy to find; Gottlob et al. (6) show that most versions of the problem of finding pure Nash equilibria are hard when the game is described succintly. Also from Fabrikant et al. (5) we know that for congestion games (where Nash equilibria minimize a potential function) it is PLS-complete to find a Nash equilibrium in general.

In contrast with Nash equilibrium, the problem of finding correlated equilibria is substantially more tractable: Papadimitriou (13) shows that for most standard forms of succinctly represented games, including graphical games, correlated equilibria can be found in polynomial time.

Lipton and Markakis (9) study the algebraic properties to the solution of a game. For a $r$-player game they exhibit a polynomial of degree $2 r$ whose roots contain the Nash equilibria, and they give algorithms for computing Nash equilibria and approximate Nash equilibria, using existing quantifier 
elimination algorithms. By applying our reduction the degree of the associated polynomial is 8 , which should lead to an improved upper bound on the time taken to find these equilibria. Note that is not an upper bound on the algebraic degree of the solutions of a game, which may be exponential in the number of actions per player, in the 4-player case.

Finally, certain other interesting reductions between equilibrium problems have been recently pointed out $(1 ; 15)$. Some of the results of $(15)$ are conceptually similar to ours, for example, Theorem 4.1 of (15) reduces graphical games to 2-strategy "circuit games", such that there are polynomial-time computable functions that map Nash equilibria from the graphical game to the corresponding circuit game, and back. (A circuit game has payoffs specified by boolean circuits that compute them, given as inputs the strategies chosen by the players.)

\subsection{Definitions-Notation}

A game $\mathcal{G}$ in normal form has $r \geq 2$ players (indexed by $p$ ) and for each player $p$ a finite set $S_{p}$ of pure strategies. The set $S$ of pure strategy profiles is the Cartesian products of the $S_{p}$ 's. The set of all strategy profiles of players other than $p$ is denoted $S_{-p}$. Finally, for each $p \leq r$ and $s \in S$ we have a payoff or utility $u_{s}^{p}$. We also use the notation $u_{j s}^{p}$ for $p \leq r, j \in S_{p}, s \in S_{-p}$.

Given real numbers $x_{j}^{p}$ for each player $p$ and strategy $j \in S_{p}$ and a strategy profile $s=\left(s_{1}, \ldots, s_{r}\right)$ where $s_{p} \in S_{p}$, we denote by $x_{s}$ the product $x_{s_{1}}^{1} \cdot x_{s_{2}}^{2} \cdots x_{s_{r}}^{r}$. Such real numbers constitute a Nash equilibrium if the following conditions hold:

$$
\begin{aligned}
& x_{j}^{p} \in[0,1] \\
& \sum_{j} x_{j}^{p}=1 \\
& \sum_{s \in S_{-p}}^{p} u_{j s}^{p} x_{s}>\sum_{s \in S_{-p}} u_{j^{\prime} s}^{p} x_{s} \Longrightarrow x_{j^{\prime}}^{p}=0 \text { for all } p, i, j \in S_{p}
\end{aligned}
$$

Intuitively, a Nash equilibrium is a probability distribution on the strategies of each player so that no player can improve its expected utility by unilaterally changing its distribution.

In a game in normal form we are given a rational number $u_{s}^{p}$ for every $p$ and $s-$ a total of $r|S|$ numbers. A graphical game $\mathcal{G G}$ is an undirected graph $G=(V, E)$, where each vertex $v$ has an associated set of strategies $S_{v}$. Let $\mathcal{N}(v)$ denote $v$ and $v$ 's neighbors in $G$, and let $S_{\mathcal{N}(v)}$ denote the set of all pure profiles of $\mathcal{N}(v)$. In a graphical game, the utilities to $v$ are given by $\left\{u_{s}^{v}: s \in S_{\mathcal{N}(v)}\right\}$. Intuitively, a graphical game is a succinct representation of a game in normal form, when it so happens that for every $p, u_{s}^{p}$ only depends on a small set of other players. Hence there is no need to redefine Nash equilibrium here. A generalization of graphical games are the directed graphical games, where $G$ is directed and $\mathcal{N}(v)$ consists of $v$ and the predecessors of $v$.

Definition 1 The (2-player) game Generalized Matching Pennies is defined as follows. Call the 2 players the pursuer and the evader, and let $[n]$ denote their strategies. If for any $i \in[n]$ both players play $i$, then the pursuer receives a positive payoff $u>0$ and the evader receives a payoff of $-u$. Otherwise both players receive 0 . It is not hard to check that the game has a unique Nash equilibrium in which both players use the uniform distribution.

We next introduce certain simple concepts from the complexity theory of total functions, see (14) for a formalism in the same spirit. A search problem $S$ is a set of inputs $I_{S}$ such that for each $x \in I_{S}$ there is an associated set of solutions $S_{x}$ (each of length bounded by a polynomial in $|x|$ ) such that for each $x \in I_{S}$ and $y \in \Sigma^{|x|^{k}}$ whether $y \in S_{x}$ is decidable in polynomial time (note that this is essentially NP, except that the emphasis is on finding a witness). For example, $r$-NASH is the search problem $S$ in which $I_{S}$ is all $r$-player games in normal form and $S_{x}$ is the set of Nash equilibria of game $x \in I_{S}$. Similarly, $d$-GRAPHICAL NASH is the search problem with inputs the 
set of all graphical games with degree at most $d$, and solutions the corresponding Nash equilibria. (Strictly speaking, since the solutions of these problems are potentially irrationally numbers, the input also includes a specification of the desired accuracy.) The search problem is total if $S_{x} \neq \emptyset$ for all $x \in I_{S}$. For example, Nash's 1951 theorem (11) implies that $r$-NASH is total. Obviously, the same is true of $d$-GRAPHICAL NASH.

A polynomially computable function $f$ is a polynomial-time reduction from total search problem $S$ to total search problem $T$ if for every input $x \in I_{S}$ of $S f(x) \in I_{T}$ is an input of $T$, and furthermore there is another polynomial function $g$ such that for every $y \in T_{f(x)}, g(y) \in S_{x}$. If furthermore for all $x g$ is an isomorphism between $T_{f(x)}$ and $S_{x}, f$ is said to be faithful. All reductions presented in this paper happen to be faithful.

\section{Reduction from Graphical Games to Normal Form Games}

Theorem 1 For every $d>1$ there is a polynomial reduction from $d$-GRAPHICAL NASH to $d^{2}$-NASH.

Input: Degree $d$ graphical game $\mathcal{G G}$, players $\left\{v_{1}, \ldots, v_{n}\right\}$ each with $t$ strategies.

Output: Normal-form game $\mathcal{G}$.

1. Re-scale the utilities $u_{j}^{p}$ so that they lie in the range $[0,1]$.

2. Let $r=d^{2}$ or $r=d^{2}-1 ; r$ chosen to be even.

3. Let $c: V \longrightarrow\{1, \ldots, r\}$ be a $r$-coloring of $G$ such that no two adjacent vertices have the same color, and furthermore no two vertices having a common neighbor have the same color. Assume the same number of vertices for each color, adding extra isolated vertices to make up any shortfall.

$$
\text { Let }\left\{v_{1}^{(i)}, \ldots, v_{n / r}^{(i)}\right\} \text { denote }\{v: c(v)=i\} .
$$

4. For $p \in[r]$ player $p$ in $\mathcal{G}$ has strategy set $S_{p}$ with $\left|S_{p}\right|=t n / r ; S_{p}$ is the union (assumed disjoint) of all $S_{v}$ with $c(v)=p$.

$$
S_{p}=\left\{\left(v_{i}, a\right): c\left(v_{i}\right)=p, a \in S_{v_{i}}\right\} .
$$

5. Let $s \in S$ be a strategy profile. For $p \in[r], u^{p}(s)$ is defined as follows:

(a) Initially, all utilities are 0 .

(b) For $v_{0} \in V$ having neighbors $v_{1}, \ldots, v_{d^{\prime}}$ in $G$, if $c\left(v_{0}\right)=p$ and for $i=0, \ldots, d^{\prime}, s$ contains $\left(v_{i}, a_{i}\right)$, then $u^{p}(s)=u^{v_{0}}\left(s^{\prime}\right)$ for $s^{\prime}$ a strategy profile of $\mathcal{G G}$ in which $v_{i}$ plays $a_{i}$ for $i=0, \ldots, d^{\prime}$.

(c) Let $M=\left(t n /\left(d^{2}-1\right)\right)^{d^{2}-1} n^{4} t r^{\prime}$.

(d) For odd number $p<2 m$, if player $p$ plays $\left(v_{i}^{(p)}, a\right)$ and $p+1$ plays $\left(v_{i}^{(p+1)}, a^{\prime}\right)$, for any $i, a, a^{\prime}$, then add $M$ to $u^{p}(s)$ and subtract $M$ from $u^{p+1}(s)$.

Figure 1: Reduction from graphical game $\mathcal{G G}$ to normal form game $\mathcal{G}$ 
Proof. Overview. Figure 1 shows the construction of $\mathcal{G}=f(\mathcal{G G})$. We show that $f$ is computable in polynomial-time, and for any Nash equilibrium $N_{\mathcal{G}}$ of $\mathcal{G}$ we can construct $N_{\mathcal{G G}}=g\left(N_{\mathcal{G}}\right)$, a Nash equilibrium of $\mathcal{G G}$.

We first rescale all payoffs so they are nonnegative and at most 1 , and assume that all vertices have the same number of strategies $\left|S_{v}\right|=t$. We then color the vertices of $G$ with at most $d^{2}-1$ colors such that any two adjacent vertices have different colors, but also no two vertices with a common neighbor have the same color. This is tantamount to coloring the union of $G$ and the square of $G$, a graph that has degree $d^{2}-1$ and is hence $d^{2}-1$-colorable. We assume for simplicity that each color class has the same number $n /\left(d^{2}-1\right)$ of vertices (adding dummy vertices if not).

We construct a normal form game $\mathcal{G}$ with $r \leq d^{2}$ players. Each player has $t n /\left(d^{2}-1\right)$ strategies, the $t$ strategies of each of the $n /\left(d^{2}-1\right)$ vertices in its color class. We can assume $r$ is even, and we divide the $r$ players into pairs, who play generalized Matching Pennies at very high stakes, so as to ensure that they will all randomize uniformly over their vertices. (A similar trick is used in Theorem 7.3 of (15), a hardness result for a class of circuit games.) Within each set of strategies associated with each vertex, the Matching Pennies game expresses no preference, and payoffs are augmented to correspond to the payoffs that would arise in the original graphical game $\mathcal{G G}$.

Polynomial size of $\mathcal{G}=f(\mathcal{G G})$.

The input size is $|\mathcal{G G}|=\Theta\left(n q \cdot t^{d+1}\right)$, where $q$ is the size of input quantities $u_{j}^{p}$ in the logarithmic cost model.

The normal form game $\mathcal{G}$ has $\leq d^{2}$ players, each having $t n /\left(d^{2}-1\right)$ strategies. Hence there are $\leq\left(\frac{t n}{d^{2}-1}\right)^{d^{2}}$ matrix entries in $\mathcal{G}$. This is polynomial so long as $d$ is constant.

The size of $M$ (in logarithmic cost) is $O\left(\left(d^{2}-1\right) \log \left(t n /\left(d^{2}-1\right)\right)\right.$ ), and all other non-zero entries of $\mathcal{G}$ are payoffs (of size $q$ ) that appear in $\mathcal{G} \mathcal{G}$.

\section{Construction of $g\left(N_{\mathcal{G}}\right)$ (where $N_{\mathcal{G}}$ denotes a Nash equilibrium of $\mathcal{G}$ ).}

Given a Nash equilibrium $N_{\mathcal{G}}$ of $f(\mathcal{G G})$, we claim we recover a Nash equilibrium of $\mathcal{G} \mathcal{G}, N_{\mathcal{G G}}=g\left(N_{\mathcal{G}}\right)$, as follows. For $t^{\prime} \in[t], x_{t^{\prime}}^{v}=x_{\left(v, t^{\prime}\right)}^{p} / \sum_{a \in[t]} x_{(v, a)}^{p}$, where $p=c(v)$. Clearly $g$ is computable in polynomial time.

\section{Proof that the reduction preserves Nash equilibria.}

For $v \in V, c(v)=p$, let " $p$ plays $v$ " denote the event that $p$ plays $(v, a)$ for some $a \in S_{v}$.

First we prove that in a Nash equilbrium $N_{\mathcal{G}}$, for every player $p$, every $v \in V$ with $c(v)=p$, $\operatorname{Pr}(p$ plays $v) \geq \frac{1}{2} r / n$. Note that the "fair share" for $v$ is $r / n$.

Suppose for a contradiction that in a Nash equilibrium of $\mathcal{G}, \operatorname{Pr}\left(p\right.$ plays $\left.v_{i}^{(p)}\right)<\frac{1}{2} r / n$. If $p$ is odd (the pursuer) then $p+1$ (the evader) will prefer $v_{i}^{(p+1)}$ to some other vertex $v_{j}^{(p+1)}$ which $p$ plays with probability $>r / n$ and which $p+1$ will play with probability zero. But if the evader is choosing certain vertices with probability zero, then there is some $v_{i}^{(p+1)}$ such that $\operatorname{Pr}\left(p+1\right.$ plays $\left.v_{i}^{(p+1)}\right)>$ $r / n$. The pursuer $p$ gets an expected payoff for playing $v_{i}^{(p)}$ of at least $M r / n$, and from playing $j$ an expected payoff of at most $1+\frac{1}{2} M / \frac{n}{r}$. The additive 1 comes from the payoffs (in [0,1]) in $\mathcal{G G}$. $M$ has been chosen large enough such that $M r / n>1+\frac{1}{2} M / \frac{n}{r}$, which contradicts the assumption that we have a Nash equilibrium.

If $p$ is even, then for some $j, p$ plays $v_{j}^{(p)}$ with probability $>r / n$. Choose $j$ such that $p$ has a non-zero probability of playing $v_{j}^{(p)}$. Then $p-1$ plays $v_{i}^{(p-1)}$ with probability 0 (since $p-1$ gets a better payoff from playing $\left.v_{j}^{(p-1)}\right)$. But then $p$ has a better payoff for playing $i$ than for $j$, a contradiction.

As a result, every vertex is chosen with probability greater than $\frac{1}{2} r / n$ by the player that represents its color class. The division of $\operatorname{Pr}(p$ plays $v)$ into $\operatorname{Pr}(p$ plays $(v, a))$ for various values of 
$a$, is driven entirely by the same payoffs as in $\mathcal{G G}$; there is some probability $p(v) \geq\left(\frac{1}{2} r / n\right)^{d}$ that the neighbors of $v$ are chosen by other players, and the additional payoff resulting from the choice of $\operatorname{Pr}(p$ plays $(v, a)), a \in[t]$, is $p(v)$ times the payoff $v$ would get in $\mathcal{G G}$.

Uniqueness of $g^{-1}\left(N_{\mathcal{G G}}\right)$.

There is a 1-1 correspondence between the Nash equilibria of $\mathcal{G}$ and $\mathcal{G} \mathcal{G}$, since every Nash equilibrium of $\mathcal{G G}$ encodes a specific Nash equilibrium of $\mathcal{G}$.

\section{Reduction from Normal Form Games to Graphical Games}

Given a normal form game $\mathcal{G}$ having $r$ players, we construct a graphical game $\mathcal{G G}$, with a bipartite underlying graph with maximum degree 3 , and two strategies per vertex, with description length polynomial in the description length of $\mathcal{G}$, so that from every Nash equilibrium of $\mathcal{G G}$ we can recover a Nash equilibrium of $\mathcal{G}$. In this subsection we decribe the basic building blocks of the construction. Each player in $\mathcal{G G}$ will have just two strategies, denoted 0 and 1 . Also, it will be easy to check that the graph of $\mathcal{G G}$ is bipartite and has degree 3 ; this graph will be denoted $G=(V \cup W, E)$, where $W$ and $V$ are disjoint, and each edge in $E$ goes between $V$ and $W$.

Notation. $\mathbf{p}[v]$ denotes the probability that $v$ plays 1 (as opposed to 0 ). $v: b$ denotes the event that the player at vertex $v$ uses strategy $b$, for $b \in\{0,1\}$.

Recall that $\mathcal{G}$ is specified by the quantities $\left\{u_{s}^{p}: p \in[r], s \in S\right\}$. A mixed strategy profile of $\mathcal{G}$ is given by probabilities $\left\{x_{j}^{p}: p \in[r], j \in[n]\right\}$. $\mathcal{G} \mathcal{G}$ will contain a vertex $v\left(x_{j}^{p}\right) \in V$ for each player $p$ and strategy $j \in S_{p}$, and the construction of $\mathcal{G G}$ will ensure that in any Nash equilibrium of $\mathcal{G G}$, the quantities $\left\{\mathbf{p}\left[v\left(x_{j}^{p}\right)\right]: p \in[r], j \in S_{p}\right\}$, if interpreted as values of $x_{j}^{p}$, will constitute a Nash equilibrium of $\mathcal{G}$. Extending this notation, for various arithmetic expressions $A$ involving any $x_{j}^{p}$ and $u_{s}^{p}$, vertex $v(A) \in V$ will be used, and be constructed such that in Nash equilibria of $\mathcal{G G}$, $\mathbf{p}[v(A)]$ is equal to $A$ evaluated at the given values of $u_{s}^{p}$ and with $x_{j}^{p}$ equal to $\mathbf{p}\left[v\left(x_{j}^{p}\right)\right]$. Elements of $W$ are used to mediate between elements of $V$, so that the latter ones obey the intended arithmetic relationships.

The following propositions show how we can ensure various arithmetic relations amongst the values $\mathbf{p}[v]$, for $v \in V$.

Proposition 1 Let $\alpha$ be a non-negative real number. Let $v_{1}, v_{2}, w$ be vertices in a graphical game $\mathcal{G G}$, and suppose that the payoffs to $v_{2}$ and $w$ are as follows.

\begin{tabular}{ll|cc} 
& & $w: 0$ & $w: 1$ \\
\hline$v_{2}: 0$ & 0 & 1 \\
$v_{2}: 1$ & 1 & 0
\end{tabular}

\begin{tabular}{|c|c|c|c|c|c|c|c|c|}
\hline \multirow{3}{*}{ Payoffs to $w:$} & \multirow{3}{*}{$w: 0$} & & $v_{2}: 0$ & $v_{2}: 1$ & \multirow[b]{2}{*}{$w: 1$} & & $v_{2}: 0$ & $v_{2}: 1$ \\
\hline & & $v_{1}: 0$ & 0 & 0 & & $v_{1}: 0$ & 0 & 1 \\
\hline & & $v_{1}: 1$ & $\alpha$ & $\alpha$ & & $v_{1}: 1$ & 0 & 1 \\
\hline
\end{tabular}

Then, in any Nash equilibrium of $\mathcal{G} \mathcal{G}, \mathbf{p}\left[v_{2}\right]=\min \left(\alpha \mathbf{p}\left[v_{1}\right], 1\right)$.

Proof. If $w$ plays 1, then the expected payoff to $w$ is $\mathbf{p}\left[v_{2}\right]$, and if $w$ plays 0 then the expected payoff to $w$ is $\alpha \mathbf{p}\left[v_{1}\right]$. Therefore, in a Nash equilibrium of $\mathcal{G G}$, if $\mathbf{p}\left[v_{2}\right]>\alpha \mathbf{p}\left[v_{1}\right]$ then $\mathbf{p}[w]=1$.

However, note also that if $\mathbf{p}[w]=1$ then $\mathbf{p}\left[v_{2}\right]=0$. (Payoffs to $v_{2}$ make it prefer to disagree with $w$.) Consequently, $\mathbf{p}\left[v_{2}\right]$ cannot be strictly larger than $\mathbf{p}\left[v_{1}\right]$.

Similarly, if $\mathbf{p}\left[v_{2}\right]<\alpha \mathbf{p}\left[v_{1}\right]$ then $\mathbf{p}[w]=0$, which implies that $\mathbf{p}\left[v_{2}\right]=1$ (again since $v_{2}$ has the biggest payoff by disagreeing with $w)$. Hence $\mathbf{p}\left[v_{2}\right]$ cannot be less than $\min \left(1, \alpha \mathbf{p}\left[v_{1}\right]\right)$. 
Note in particular that using the above construction with $\alpha=1, v_{2}$ becomes a "copy" of $v_{1}$ and we can make a sequence of copies of any vertex, which form a path in the graph. The copies alternate with distinct $w$ vertices.

Proposition 2 Let $\alpha, \beta, \gamma$ be non-negative real numbers. Let $v_{1}, v_{2}, v_{3}, w$ be vertices in a graphical game $\mathcal{G G}$, and suppose that the payoffs to $v_{3}$ and $w$ are as follows.

$$
\begin{array}{lc|cc} 
& & w: 0 & w: 1 \\
\hline \text { Payoffs to } v_{3}: & v_{3}: 0 & 0 & 1 \\
v_{3}: 1 & 1 & 0
\end{array}
$$

\begin{tabular}{lll|cccc|c} 
& & & $v_{2}: 0$ & $v_{2}: 1$ & & & \\
\cline { 2 - 5 } Payoffs to $w:$ & $w: 0$ & & $w: 1$ & $v_{3}: 0$ & 0 \\
& & $v_{1}: 0$ & 0 & $\beta$ & & & \\
& $v_{1}: 1$ & $\alpha$ & $\alpha+\beta+\gamma$ & & & $v_{3}: 1$ & 1
\end{tabular}

Then, in any Nash equilibrium of $\mathcal{G G}, \mathbf{p}\left[v_{2}\right]=\min \left(\alpha \mathbf{p}\left[v_{1}\right]+\beta \mathbf{p}\left[v_{2}\right]+\gamma \mathbf{p}\left[v_{1}\right] \mathbf{p}\left[v_{2}\right], 1\right)$.

Proof. If $w$ plays 1 , then the expected payoff to $w$ is $\mathbf{p}\left[v_{3}\right]$, and if $w$ plays 0 then the expected payoff to $w$ is $\alpha \mathbf{p}\left[v_{1}\right]+\beta \mathbf{p}\left[v_{2}\right]+\gamma \mathbf{p}\left[v_{1}\right] \mathbf{p}\left[v_{2}\right]$. Therefore, in a Nash equilibrium of $\mathcal{G G}$, if $\mathbf{p}\left[v_{3}\right]>$ $\alpha \mathbf{p}\left[v_{1}\right]+\beta \mathbf{p}\left[v_{2}\right]+\gamma \mathbf{p}\left[v_{1}\right] \mathbf{p}\left[v_{2}\right]$ then $\mathbf{p}[w]=1$.

However, note from the payoffs to $v_{3}$ that if $\mathbf{p}[w]=1$ then $\mathbf{p}\left[v_{3}\right]=0$. Consequently, $\mathbf{p}\left[v_{3}\right]$ cannot be strictly larger than $\alpha \mathbf{p}\left[v_{1}\right]+\beta \mathbf{p}\left[v_{2}\right]+\gamma \mathbf{p}\left[v_{1}\right] \mathbf{p}\left[v_{2}\right]$.

Similarly, if $\mathbf{p}\left[v_{3}\right]<\alpha \mathbf{p}\left[v_{1}\right]+\beta \mathbf{p}\left[v_{2}\right]+\gamma \mathbf{p}\left[v_{1}\right] \mathbf{p}\left[v_{2}\right]$ then due to the payoffs to $w$ we have $\mathbf{p}[w]=0$. This in turn implies that $\mathbf{p}\left[v_{3}\right]=1$ (since $v_{3}$ has the biggest payoff by disagreeing with $w$ ). Hence $\mathbf{p}\left[v_{3}\right]$ cannot be less than $\min \left(1, \alpha \mathbf{p}\left[v_{1}\right]+\beta \mathbf{p}\left[v_{2}\right]+\gamma \mathbf{p}\left[v_{1}\right] \mathbf{p}\left[v_{2}\right]\right)$.

Using the above construction, we can compute sums and products of quantities represented as probabilities that particular vertices choose to play strategy 1 . In particular, if a vertex $v\left(x_{j}^{p}\right)$ encodes probability $x_{j}^{p}$, then we can compute the expressions $\sum_{s \in S_{-p}} u_{j s}^{p} x_{s}$. The challenge is now to allow the values to feed back to the vertices $v\left(x_{j}^{p}\right)$ encoding the $x_{j}^{p}$ values, the constraint $\sum_{s \in S_{-p}} u_{j s}^{p} x_{s}>\sum_{s \in S_{-p}} u_{j^{\prime} s}^{p} x_{s} \Longrightarrow x_{j^{\prime}}^{p}=0$.

Proposition 3 Let $v_{1}, v_{2}, v_{3}, v_{4}, v_{5}, v_{6}, w_{1}, w_{2}, w_{3}, w_{4}$ be vertices in a graphical game $\mathcal{G G}$, and suppose that the payoffs to vertices other than $v_{1}$ and $v_{2}$ are as follows.

\begin{tabular}{|c|c|c|c|c|c|c|c|c|c|}
\hline \multirow{3}{*}{ Payoffs to $w_{1}$ : } & \multirow{3}{*}{$w_{1}: 0$} & & $v_{2}:($ & $v_{2}: 1$ & \multirow{2}{*}{\multicolumn{2}{|c|}{$w_{1}: 1$}} & & \multirow{2}{*}{$\frac{v_{2}: 0}{0}$} & $v_{2}: 1$ \\
\hline & & $\overline{v_{1}: 0}$ & 0 & 0 & & & $\overline{v_{1}: 0}$ & & 1 \\
\hline & & $v_{1}: 1$ & 1 & 1 & & & $v_{1}: 1$ & 0 & 1 \\
\hline & \multirow{3}{*}{\multicolumn{2}{|c|}{ Payoffs to $v_{5}$}} & & & $w_{1}: 0$ & $w_{1}: 1$ & & & \\
\hline & & & & $v_{5}: 0$ & 1 & 0 & & & \\
\hline & & & & $v_{5}: 1$ & 0 & 1 & & & \\
\hline
\end{tabular}

Payoffs to $w_{2}$ and $v_{3}$ are chosen using Proposition 2 to ensure $\mathbf{p}\left[v_{3}\right]=\mathbf{p}\left[v_{1}\right]\left(1-\mathbf{p}\left[v_{5}\right]\right)$.

Payoffs to $w_{3}$ and $v_{4}$ are chosen using Proposition 2 to ensure $\mathbf{p}\left[v_{4}\right]=\mathbf{p}\left[v_{2}\right] \mathbf{p}\left[v_{5}\right]$.

Payoffs to $w_{4}$ and $v_{6}$ are chosen using Proposition 2 to ensure $\mathbf{p}\left[v_{6}\right]=\min \left(1, \mathbf{p}\left[v_{3}\right]+\mathbf{p}\left[v_{4}\right]\right)$.

Then, in any Nash equilibrium of $\mathcal{G G}, \mathbf{p}\left[v_{6}\right]=\max \left(\mathbf{p}\left[v_{1}\right], \mathbf{p}\left[v_{2}\right]\right)$.

It is actually possible to "merge" $w_{1}$ and $v_{5}$ in the above, but then the graph would not be bipartite. 
Proof. If, in a Nash equilibrium, we have $\mathbf{p}\left[v_{1}\right]<\mathbf{p}\left[v_{2}\right]$ then it follows from $w_{1}$ 's payoffs that $\mathbf{p}\left[w_{1}\right]=1$. It then follows that $\mathbf{p}\left[v_{5}\right]=1$ since $v_{5}$ 's payoffs induce it to imitate $w_{1}$. Hence, $\mathbf{p}\left[v_{3}\right]=0$ and $\mathbf{p}\left[v_{4}\right]=\mathbf{p}\left[v_{2}\right]$, and consequently, $\mathbf{p}\left[v_{5}\right]=\mathbf{p}\left[v_{4}\right]=\mathbf{p}\left[v_{2}\right]$, as required. A similar argument shows that if $\mathbf{p}\left[v_{1}\right]>\mathbf{p}\left[v_{2}\right]$ then $\mathbf{p}\left[v_{5}\right]=\mathbf{p}\left[v_{1}\right]$.

If $\mathbf{p}\left[v_{1}\right]=\mathbf{p}\left[v_{2}\right]$ then $\mathbf{p}\left[w_{1}\right]$ may take any value. However, we have

$$
\begin{aligned}
\mathbf{p}\left[v_{3}\right] & =\mathbf{p}\left[v_{1}\right]\left(1-\mathbf{p}\left[v_{5}\right]\right) \\
\mathbf{p}\left[v_{4}\right] & =\mathbf{p}\left[v_{2}\right] \mathbf{p}\left[v_{5}\right]=\mathbf{p}\left[v_{1}\right] \mathbf{p}\left[v_{5}\right]
\end{aligned}
$$

Finally,

$$
\begin{aligned}
\mathbf{p}\left[v_{6}\right] & =\min \left(1, \mathbf{p}\left[v_{3}\right]+\mathbf{p}\left[v_{4}\right]\right) \\
& =\min \left(1, \mathbf{p}\left[v_{1}\right]\left(1-\mathbf{p}\left[w_{1}\right]\right)+\mathbf{p}\left[v_{1}\right] \mathbf{p}\left[w_{1}\right]\right) \\
& =\mathbf{p}\left[v_{1}\right]
\end{aligned}
$$

We use Propositions (1-3) as building blocks of $\mathcal{G G}$, starting with $r$ subgraphs that represent mixed strategies for the players of $\mathcal{G}$. In the following we make a binary tree with leaves $v\left(x_{j}^{p}\right)$ whose probabilities sum to 1 , and internal nodes $y_{j}^{p}$ which use their probabilities to select subtrees (see Figure 2 for an illustration).

Proposition 4 Consider a graphical game that contains

- for $j \in[n]$ a vertex $v\left(x_{j}^{p}\right)$

- for $j \in[n-1]$ a vertex $v_{j}^{p}$

- for $j \in[n]$ a vertex $v\left(\sum_{i=1}^{j} x_{i}^{p}\right)$

- for $j \in[n-1]$ a vertex $w_{j}(p)$ used to ensure $\mathbf{p}\left[v\left(\sum_{i=1}^{j} x_{i}^{p}\right)\right]=\mathbf{p}\left[v\left(\sum_{i=1}^{j+1} x_{i}^{p}\right)\right]\left(1-\mathbf{p}\left[v_{j}^{p}\right]\right)$

- for $j \in[n-1]$ a vertex $w_{j}^{\prime}(p)$ used to ensure $\mathbf{p}\left[v\left(x_{j+1}^{p}\right)\right]=\mathbf{p}\left[v\left(\sum_{i=1}^{j+1} x_{i}^{p}\right)\right] \mathbf{p}\left[v_{j}^{p}\right]$

- a vertex $w_{0}^{\prime}(p)$ used to ensure $\mathbf{p}\left[v\left(x_{1}^{p}\right)\right]=\mathbf{p}\left[v\left(\sum_{i=1}^{1} x_{i}^{p}\right)\right]$

$v\left(\sum_{i=1}^{n} x_{i}^{p}\right)$ has payoff 1 when it plays 1 , 0 otherwise.

Then $\sum_{i=1}^{n} \mathbf{p}\left[v\left(x_{i}^{p}\right)\right]=1$ and moreover $\mathbf{p}\left[v\left(\sum_{i=1}^{j} x_{i}^{p}\right)\right]=\sum_{i=1}^{j} \mathbf{p}\left[v\left(x_{i}^{p}\right)\right]$, and the graph is bipartite and of degree 3 .

Proof. It is not hard to verify that the graph has degree 3. Most of the degree 3 nodes are the $w$ nodes used in Propositions 1 and 2 to connect the pairs or triples of graph players whose probabilities are supposed to obey an arithmetic relationship.

In a Nash equilibrium, $v\left(\sum_{i=1}^{n} x_{i}^{p}\right)$ plays 1 . The vertices $v_{j}^{p}$ split this probability into the two subtrees below them.

Comment. The values $\mathbf{p}\left[v_{j}^{p}\right]$ control the distribution of probability (summing to 1 ) amongst the $n$ vertices $v\left(x_{j}^{p}\right)$. These vertices can set to zero any proper subset of the probabilities $\mathbf{p}\left[v\left(x_{j}^{p}\right)\right]$.

Notation. For $s \in S_{-p}$ let $x_{s}=x_{s_{1}}^{1} \cdot x_{s_{2}}^{2} \cdots x_{s_{p-1}}^{p-1} \cdot x_{s_{p+1}}^{p+1} \cdots x_{s_{r}}^{r}$. For $s \in S_{-p}$ let $U_{j}^{p}=\sum_{s \in S_{-p}} u_{j s}^{p} x_{s}$. Thus $U_{j}^{p}$ is the utility to $p$ of playing $j$ in the context of a given mixed profile. 


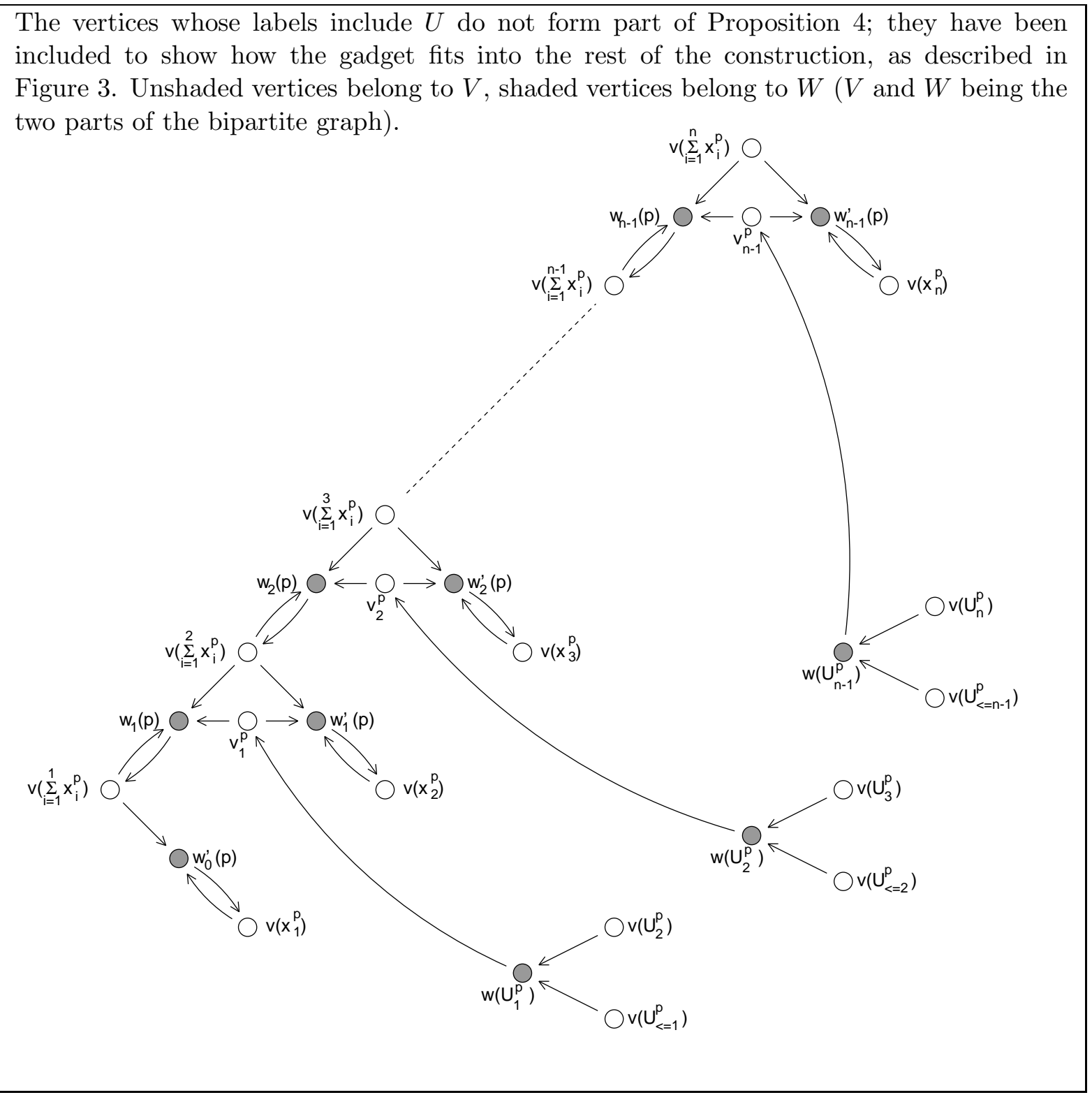

Figure 2: Diagram of Proposition 4

Lemma 1 Suppose all utilities $u_{s}^{p}$ lie in the range $[0,1]$. We can construct a degree 3 bipartite graph having a total of $O\left(r n^{r-1}\right)$ vertices, including vertices $v\left(x_{j}^{p}\right), v\left(U_{j}^{p}\right), v\left(U_{\leq j}^{p}\right)$, for $p \in[r]$, $j \in[n]$, such that in any Nash equilibrium,

$$
\mathbf{p}\left[v\left(U_{j}^{p}\right)\right]=\sum_{s \in S_{-p}} u_{j s}^{p} \prod_{\ell \neq p} \mathbf{p}\left[v\left(x_{s_{\ell}}^{\ell}\right)\right] \quad \mathbf{p}\left[v\left(U_{\leq j}^{p}\right)\right]=\max _{i \leq j} \sum_{s \in S_{-p}} u_{j s}^{p} \prod_{\ell \neq p} \mathbf{p}\left[v\left(x_{s_{\ell}}^{\ell}\right)\right]
$$

The general idea is to note that the ezxpressions for $\mathbf{p}\left[v\left(U_{j}^{p}\right)\right]$ and $\mathbf{p}\left[v\left(U_{\leq j}^{p}\right)\right]$ are constructed from arithmetic subexpressions using the operations of addition, multiplication and maximization. If each subexpression $E$ has a vertex $v(E)$, then using Propositions 2 and 3 we can assemble them into a graphical game such that in any Nash equilibrium, $\mathbf{p}[v(e)]$ is equal to the value of $E$ with 
input $\mathbf{p}\left[v\left(x_{j}^{p}\right)\right], p \in[r], j \in[n]$. We just need to limit our usage to $O\left(r n^{r}\right)$ subexpressions and ensure that their values all lie in $[0,1]$.

Proof.

$$
\begin{gathered}
U_{\leq j}^{p}=\max \left\{U_{j}^{p}, U_{\leq j-1}^{p}\right\}, \quad U_{j}^{p}=\sum_{s \in S_{-p}} u_{j s}^{p} x_{s} \\
\sum_{s \in S_{-p}} u_{j s}^{p} x_{s}=\sum_{s \in S_{-p}} u_{j s}^{p} x_{s_{1}}^{1} \cdots x_{s_{p-1}}^{p-1} x_{s_{p+1}}^{p+1} \cdots x_{s_{r}}^{r}
\end{gathered}
$$

Let $S_{-p}=\left\{S_{-p}(1), \ldots, s_{-p}\left(n^{r-1}\right)\right\}$.

$$
\sum_{s \in S_{-p}} u_{j s}^{p} x_{s}=\sum_{\ell=1}^{n^{r-1}} u_{j s}^{p} S_{-p}(\ell)
$$

For each partial sum $\sum_{\ell=1}^{z} u_{j s}^{p} S_{-p}(\ell)$ include vertex $v\left(\sum_{\ell=1}^{z} u_{j s}^{p} S_{-p}(\ell)\right)$.

Similarly for each partial product of the summands $u_{j s}^{p} x_{s_{1}}^{1} \cdots x_{s_{z}}^{z}$ include vertex $v\left(u_{j s}^{p} x_{s_{1}}^{1} \cdots x_{s_{z}}^{z}\right)$

There are $p$ partial sums for each summand. There are $n^{r-1}$ partial sums. There are $p$ partial sequences over which we have to maximize. So using a vertex for each of $2 p+n^{r-1}$ arithmetic subexpressions, a Nash equilibrium will compute the desired quantities. All of these quantities in the subexpressions lie in the range $[0,1]$, so the ceiling of 1 in the computations of Propositions 1,2,3 is not a problem.

Theorem 2 For any fixed $r>1$, there is a polynomial reduction from $r$-NASH to 3 -GRAPHICAL NASH with two strategies per vertex.

Proof. Let $\mathcal{G}$ be a $r$-player normal-form game, and construct $\mathcal{G G}$ from $\mathcal{G}$ as shown in Figure 3 . The graph has degree 3 , since we use separate copies of the $v\left(x_{j}^{p}\right)$ vertices to influence different $v\left(U_{j}^{p}\right)$ vertices.

Polynomial size of $\mathcal{G G}=f(\mathcal{G})$.

The size of $\mathcal{G G}$ is proportional to the description length $r \cdot n^{r}$ of $\mathcal{G}$ (using the standard representation).

Construction of $g\left(N_{\mathcal{G G}}\right)$ (where $N_{\mathcal{G G}}$ denotes a Nash equilibrium of $\mathcal{G G}$ ).

$g\left(N_{\mathcal{G G}}\right)$ is defined by letting $x_{j}^{p}=\mathbf{p}\left[v\left(x_{j}^{p}\right)\right]$, clearly computable in polynomial-time.

\section{Proof that the reduction preserves Nash equilibria.}

We show that any Nash equilibrium $N_{\mathcal{G G}}$ of $\mathcal{G G}$ has a corresponding $g\left(N_{\mathcal{G G}}\right)$, Nash equilibrium of $\mathcal{G}$.

By Proposition 4 and Lemma 1, we have the first two parts of (1). We need to show the last part, i.e. that $\sum_{s \in S_{-p}} u_{j s}^{p} x_{s}>\sum_{s \in S_{-p}} u_{j^{\prime} s}^{p} x_{s} \Longrightarrow x_{j^{\prime}}^{p}=0$.

Suppose that $\sum_{s \in S_{-p}} u_{j s}^{p} x_{s}>\sum_{s \in S_{-p}} u_{j^{\prime} s}^{p} x_{s}$. Suppose first that $j<j^{\prime}$. Then $\mathbf{p}\left[v\left(U_{\leq j-1}^{p}\right)\right]>$ $\mathbf{p}\left[v\left(U_{j}^{p}\right)\right]$, so $\mathbf{p}\left[v_{j-1}^{p}\right]=0$ and consequently $v\left(x_{j}^{p}\right)$ plays 0 as required (since $\left.\mathbf{p}\left[v\left(x_{j}^{p}\right)\right]=\mathbf{p}\left[v_{j-1}^{p}\right] \mathbf{p}\left[v\left(\sum_{i=1}^{j} x_{i}^{p}\right)\right]\right)$. If $j>j^{\prime}$, consider $\mathbf{p}\left[v\left(U_{\leq j-1}^{p}\right)\right]$. If $\mathbf{p}\left[v\left(U_{\leq j-1}^{p}\right)\right]<\mathbf{p}\left[v\left(U_{j}^{p}\right)\right]$ then $\mathbf{p}\left[v_{j-1}^{p}\right]=1$ and for all $i<j$ we get as a result $\mathbf{p}\left[v\left(x_{i}^{p}\right)\right]=0$ (since these quantities are multiples of $\left.1-\mathbf{p}\left[v_{j-1}^{p}\right]\right)$. If $\mathbf{p}\left[v\left(U_{\leq j-1}^{p}\right)\right] \geq$ $\mathbf{p}\left[v\left(U_{j}^{p}\right)\right]$ then either $\mathbf{p}\left[v\left(U_{j-1}^{p}\right)\right] \geq \mathbf{p}\left[v\left(U_{j}^{p}\right)\right]$ (in which case, use $j-1$ instead of $j$ ) or $\mathbf{p}\left[v\left(U_{j-1}^{p}\right)\right]<$ $\mathbf{p}\left[v\left(U_{j}^{p}\right)\right]$, in which case $\mathbf{p}\left[v\left(U_{\leq j-2}^{p}\right)\right]=\mathbf{p}\left[v\left(U_{\leq j-1}^{p}\right)\right]$, so again use $j-1$ instead of $j$.

Uniqueness of $g^{-1}\left(N_{\mathcal{G}}\right)$.

We have shown by construction of $\mathcal{G G}$, that any Nash equilibrium forces all the quantities $\mathbf{p}\left[v\left(x_{j}^{p}\right)\right]$ to obey the constraints (1). To show the reduction is faithful, we also show that given a Nash 
Input: Normal form game $\mathcal{G}$ having $r$ players, $n$ strategies per player, given by utilities $\left\{u_{s}^{p}: p \in[r], s \in S\right\}$

Output: graphical game $\mathcal{G G}$ with bipartite graph $(V \cup W, E)$.

1. Re-scale the utilities $u_{s}^{p}$ so that they lie in the range $[0,1]$.

2. For each player/strategy pair $(p, j)$ let $v\left(x_{j}^{p}\right) \in V$ be a vertex in $\mathcal{G G}$.

3. For each $p \in[r]$ construct a subgraph as described in Proposition 4 so that in a Nash equilibrium of $\mathcal{G G}$, we have $\sum_{j} \mathbf{p}\left[v\left(x_{j}^{p}\right)\right]=1$.

4. Use the construction of Proposition 1 with $\alpha=1$ to make $n r$ copies of the $v\left(x_{j}^{p}\right)$ vertices (which are added to $V$ ).

5. Use the construction of Lemma 1 to introduce (add to $V$ ) vertices $v\left(U_{j}^{p}\right), v\left(U_{\leq j}^{p}\right)$, for all $p \in[r], j \in[n]$. Each $v\left(U_{j}^{p}\right)$ uses its own set of copies of the vertices $v\left(x_{j}^{\bar{p}}\right)$. For $p \in[r], j \in[n]$ introduce (add to $W$ ) $w\left(U_{j}^{p}\right)$ with

(a) If $w\left(U_{j}^{p}\right)$ plays 0 then $w\left(U_{j}^{p}\right)$ gets payoff 1 whenever $v\left(U_{\leq j}^{p}\right)$ plays 1 , else 0 .

(b) If $w\left(U_{j}^{p}\right)$ plays 1 then $w\left(U_{j}^{p}\right)$ gets payoff 1 whenever $v\left(U_{j+1}^{p}\right)$ plays 1 , else 0 .

6. Give the following payoffs to the vertices $v_{j}^{p}$ (the additional vertices used in Proposition 4 whose payoffs were not specified).

(a) If $v_{j}^{p}$ plays 0 then $v_{j}^{p}$ has a payoff of 1 whenever $w\left(U_{j}^{p}\right)$ plays 0 , otherwise 1 .

(b) If $v_{j}^{p}$ plays 1 then $v_{j}^{p}$ has a payoff of 1 whenever $w\left(U_{j}^{p}\right)$ plays 1 , otherwise 0 .

Figure 3: Reduction from normal form game $\mathcal{G}$ to graphical game $\mathcal{G G}$

equilibrium $N_{\mathcal{G}}$ of $\mathcal{G}$ there is a unique corresponding Nash equilibrium $N_{\mathcal{G G}}$ of $\mathcal{G G}$, where $N_{\mathcal{G}}=$ $g^{-1}\left(N_{\mathcal{G}}\right)$.

Let $N_{\mathcal{G}}=\left\{x_{j}^{p}: p \in[r], j \in S_{p}\right\}$. In $N_{\mathcal{G G}}$, let $\mathbf{p}\left[v\left(x_{j}^{p}\right)\right]=x_{j}^{p}$.

Lemma 1 shows that the values $\mathbf{p}\left[v\left(U_{j}^{p}\right)\right]$ are the expected utilities to player $p$ of strategy $j$, given that all other players use the mixed strategy $\left\{x_{j}^{p}: p \in[r], j \in S_{p}\right\}$.

We identify values for $\mathbf{p}\left[v_{j}^{p}\right]$ that complete a Nash equilibrium for $\mathcal{G} \mathcal{G}$.

Based on the payoffs to $v_{j}^{p}$ described in Figure 3 we have

- If $\mathbf{p}\left[v\left(U_{\leq j}^{p}\right)\right]>\mathbf{p}\left[v\left(U_{j+1}^{p}\right)\right]$ then $\mathbf{p}\left[w\left(U_{j}^{p}\right)\right]=0 ; \mathbf{p}\left[v_{j}^{p}\right]=0$

- If $\mathbf{p}\left[v\left(U_{\leq j}^{p}\right)\right]<\mathbf{p}\left[v\left(U_{j+1}^{p}\right)\right]$ then $\mathbf{p}\left[w\left(U_{j}^{p}\right)\right]=1 ; \mathbf{p}\left[v_{j}^{p}\right]=1$

- If $\mathbf{p}\left[v\left(U_{\leq j}^{p}\right)\right]=\mathbf{p}\left[v\left(U_{j+1}^{p}\right)\right]$ then choose $\mathbf{p}\left[w\left(U_{j}^{p}\right)\right]=\frac{1}{2} ; \mathbf{p}\left[v_{j}^{p}\right]$ is arbitrary (we may assign it any value)

Given the above constraints on the values $\mathbf{p}\left[v_{j}^{p}\right]$ we must check that we can choose them (and there is a unique choice) so as to make them consistent with the probabilities $\mathbf{p}\left[v\left(x_{j}^{p}\right)\right]$. We use the fact the values $x_{j}^{p}$ form a Nash equilibrium of $\mathcal{G}$. In particular, we know that $\mathbf{p}\left[v\left(x_{j}^{p}\right)\right]=0$ if there exists $j^{\prime}$ with $U_{j^{\prime}}^{p}>U_{j}^{p}$. 
We claim that for $j$ satisfying $\mathbf{p}\left[v\left(U_{\leq j}^{p}\right)\right]=\mathbf{p}\left[v\left(U_{j+1}^{p}\right)\right]$, if we choose

$$
\mathbf{p}\left[v_{j}^{p}\right]=\sum_{i=1}^{j} \mathbf{p}\left[v\left(x_{i}^{p}\right)\right] / \sum_{i=1}^{j+1} \mathbf{p}\left[v\left(x_{i}^{p}\right)\right],
$$

then the values $\mathbf{p}\left[v\left(x_{j}^{p}\right)\right]$ are consistent.

\section{Combining the Reductions}

Suppose that we take either a graphical or a normal-form game, and apply to it both of the reductions described in the previous sections. Then we obtain a game of the same type that is at least as hard to solve, despite having certain restrictions on its structure. We obtain the following results.

Corollary 1 If it is possible in polynomial time to solve a graphical game having 2 strategies per player, and an underlying graph that is bipartite and of degree 3, then it is possible in polynomial time to solve a graphical game with $t$ strategies per player on any degree $d$ graph, for $d$ and $t$ constant.

The following also follows directly from Theorems 2 and 1, but is not as strong as Theorem 3 below.

Corollary 2 For any fixed $r>1$, there is a polynomial reduction from $r$-NASH to 8-NASH.

Proof. Theorem 2 converts a $r$-player game $\mathcal{G}$ into a graphical game $\mathcal{G G}$ with $d=3$. Theorem 1 converts $\mathcal{G G}$ into a 8 -player game $\mathcal{G}^{\prime}$, where $8=d^{2}-1$ whose Nash equilibria encode the Nash equilibria of $\mathcal{G G}$ and hence of $\mathcal{G}$. (Note that for $d$ an odd number, the proof of Theorem 1 implies a reduction to $d^{2}-1$-NASH; for $d$ even it reduces to $d^{2}$-NASH.)

We prove Theorem 3 below, by exploiting in more detail the structure of games $\mathcal{G G}$ constructed by Theorem 2 . The following definition is used in this respect.

Definition 2 Suppose that $\mathcal{G G}$ is a graphical game with underlying graph $G=(V, E)$. The affectsgraph $G^{\prime}=\left(V, E^{\prime}\right)$ of $\mathcal{G G}$ is a directed graph with edge $\left(v_{1}, v_{2}\right) \in E^{\prime}$ if the payoff to $v_{2}$ depends partly on the action of $v_{1}$.

Thus the edge $\left(v_{1}, v_{2}\right)$ in $G^{\prime}$ represents the relationship " $v_{1}$ affects $v_{2}$ ". Notice that if $\left(v_{1}, v_{2}\right) \in E^{\prime}$ then $\left\{v_{1}, v_{2}\right\} \in E$.

Theorem 3 For any fixed $r>1$, there is a polynomial reduction from $r$-NASH to 4 -NASH.

Proof. Construct $\mathcal{G}^{\prime}$ from $\mathcal{G}$ as shown in Figure 4.

Polynomial size of $\mathcal{G}^{\prime}=f(\mathcal{G})$.

By Theorem 2, $\mathcal{G G}$ (as constructed in Figure 4) is polynomial size. The size of $\mathcal{G} \mathcal{G}^{\prime}$ is at most 3 times the size of $\mathcal{G G}$ since we do not need to apply Step 3 to any edges that are themselves constructed by an earlier iteration of Step 3. Finally it is straightforward to check that the size of $\mathcal{G}^{\prime}$ is linear in the size of $\mathcal{G G}^{\prime}$. 
Construction of $g\left(N_{\mathcal{G}^{\prime}}\right)$ (for $N_{\mathcal{G}^{\prime}}$ a Nash equilibrium of $\mathcal{G}^{\prime}$ ).

For each $j$, each $p$, there is a probability at least $(1 / 4 N)^{4}$ that $p$ plays $\{2 j, 2 j+1\}$ and the other players play the vertices that affect $v_{p}^{(j)}$. We claim that the values $\operatorname{Pr}(p$ plays $2 j+1) /(\operatorname{Pr}(p$ plays $j)+$ $\operatorname{Pr}(p$ plays $2 j+1))$, if used for $\mathbf{p}\left[v_{p}^{(j)}\right]$, make a Nash equilibrium of $\mathcal{G G}^{\prime}$. The Nash equilibria of $\mathcal{G} \mathcal{G}^{\prime}$ and $\mathcal{G G}$ are in a 1-1 correspondence that is clear from the construction of $\mathcal{G G}^{\prime}$. Finally we recover a Nash equilibrium of $\mathcal{G}$ using the function $g$ of Theorem 2.

Proof that the reduction preserves Nash equilibria.

Let $N_{\mathcal{G}^{\prime}}$ be a Nash equilibrium of the 4-player game $\mathcal{G}^{\prime}$. We must show that we do indeed get a Nash equilibrium of $\mathcal{G G}$ if we use the above formula.

We prove the following claim (that shows that the denominator is non-zero).

Claim. For $p \in[4], j \in[N]$,

$$
\operatorname{Pr}(p \text { plays } 2 j)+\operatorname{Pr}(p \text { plays } 2 j+1) \geq \frac{1}{2 N} .
$$

Proof. Suppose otherwise, i.e. for some $p, j$,

$$
\operatorname{Pr}(p \text { plays } 2 j)+\operatorname{Pr}(p \text { plays } 2 j+1)<\frac{1}{2 N} .
$$

Let $p^{\prime}$ be $p$ 's opponent in the generalized matching pennies game. If $p^{\prime}$ is the pursuer then $p^{\prime}$ will not play $\{2 j, 2 j+1\}$ since if $p^{\prime}$ transfers $\epsilon$ from $\{2 j, 2 j+1\}$ to elsewhere he can increase his payoff, and the payoffs that encode $\mathcal{G G}^{\prime}$ are overwhelmed by the $M$ payoffs. Hence $p$ plays $2 j$ or $2 j+1$ for values of $j$ which $p^{\prime}$ plays with probability less than $\frac{1}{4 N}$; not a Nash equilibrium. If $p^{\prime}$ is the evader then $p^{\prime}$ will play $2 j$ or $2 j+1$ for values of $j$ for which $p$ 's probability of playing $\{j, j+1\}$ is less than $\frac{3}{4 N}$, again not a Nash equilibrium.

Let $q \geq(1 / 4 N)^{4}$ denote the above probability. If in $\mathcal{G G}^{\prime}$, the expected utility to $v_{p}^{(j)}$ is $u_{b}$ if $v_{p}^{(j)}$ plays $b$, then in the 4-player game, if $p$ uses $\operatorname{Pr}(p: 2 j+b) /(\operatorname{Pr}(2 p: j)+\operatorname{Pr}(p: 2 j+1))=1$ his expected payoff increases by $q u_{b}$. There is no other factor influencing $p$ 's choice about how to share the probability he plays $\{2 j, 2 j+1\}$, into the two alternatives $2 j$ and $2 j+1$. So the Nash equilibria correspond.

Let $G=(V \cup W, E)$ be the affects-graph of $\mathcal{G G}(V, W$ as defined in Figure 3). By construction of $\mathcal{G G}$

- every $W$-vertex has at most 3 incoming edges (from $v$-vertices)

- every $V$-vertex has at most 1 incoming edge and 2 outgoing edges (to/from $w$-vertices)

- every $W$-vertex has $\leq 1$ outgoing edge

Let $G^{\prime}=\left(V^{\prime} \cup W^{\prime}, E^{\prime}\right)$ be the affects-graph of $\mathcal{G G}^{\prime}$ constructed in Step 3. The payoffs in $\mathcal{G G}^{\prime}$ respect the structure of the new affects-graph (a vertex is affected by another only if there an edge between them in the graph.) All other aspects of any Nash equilibrium are preserved.

At this point we have a 4-coloring with the feature that the $V$-vertices have incoming edges from vertices of distinct colors (since they have only one incoming edge).

In Step 5 we pair off players 1 and 2, and players 3 and 4 into two independent games of generalized Matching Pennies, and all players will randomize uniformly over pairs $\{2 j, 2 j+1\}$. Next we add payoffs that encode $\mathcal{G} \mathcal{G}^{\prime}$.

In $\mathcal{G G}$ and hence $\mathcal{G G}^{\prime}$, payoffs are rescaled to lie in $[0,1]$. This fact is used to argue that the $M$ payoffs in Step 5 are large enough that, despite the modifications to the payoffs, no player gives probability 0 to any pair of actions $\{2 j, 2 j+1\}$. 


\section{Some Corollaries}

We mentioned in Section 1.1 that the solution(s) of a 4-player game may have degree exponential in the number of strategies available to the number of players. To see this, constuct a graphical game as follows. Let $x=\mathbf{p}[v]$, the probability that some vertex $v$ plays 1 . Using the gadgets we introduced in Propositions $(1,2)$, we can construct a vertex $v^{\prime}$ such that $\mathbf{p}\left[v^{\prime}\right]=x^{N}$, where $N$ is exponential in the number of vertices (this is done mainly with repeated squaring; make a copy of a vertex and multiply the two copies). Then, if $\mathbf{p}\left[v^{\prime}\right]>\frac{1}{2}$, we can give $v$ an incentive to reduce $\mathbf{p}[v]$, and if $\mathbf{p}\left[v^{\prime}\right]<\frac{1}{2}$ we give $v$ an incentive to increase $\mathbf{p}[v]$. As a result, in any Nash equilibrium, $\mathbf{p}[v]=\left(\frac{1}{2}\right)^{1 / N}$. Datta (4) shows that a 3 -player game may have solutions whose algebraic degree is linear in the number of strategies per player; we believe it is an open question whether the degree could be much higher in the 3-player case.

Suppose we are interested in solving games on a graph with a 2-dimensional grid topology. Daskalakis and Papadimitriou (3) consider grid graphs where all players are the same (having the same number of strategies, and payoffs in terms of their strategies and their neighbors' strategies). They show that the problem of finding pure Nash equilibrium is in $\mathrm{P}$ in one dimension, and NEXPcomplete in more than one dimension. Let us suppose instead that each vertex has its payoffs specified in terms of its neighbors in an ad-hoc fashion, which makes the description size much less succinct, in particular it is proportional to the number of vertices. We claim that under this restriction, the problem of finding an unrestricted Nash equilibrium remains equivalent to general low-degree graphs.

We may use Proposition 1 with $\alpha=1$ to build paths that transport values around the grid, and then what we need is a gadget that allows two of these paths to cross. See Figure 5, which copies a value $x$ from $(-3,0)$ over to $(1,0)$, and copies $y$ from $(0,-3)$ to $(0,3)$. The $W$-vertices are shaded, and do the job of computing arithmetic relationships amongst their neighbors, as indicated by their subscripts. Outgoing edges from $W$-vertices point to the vertices whose values are being computed, based on the other neighbors of the $W$-vertex.

Acknowledgements. We would like to thank Edith Elkind for informative discussions on other research related to this paper.

\section{References}

[1] B. Codenotti, A. Saberi, K. Varadarajan, Y. Ye "Leontief Economies Encode Nonzero Sum Two-Player Games," manuscript available at ECCC.

[2] V. Conitzer and T. Sandholm. "Complexity Results about Nash Equilibria", Proceedings of 18th IJCAI, pp. 765-771, Acapulco, Mexico, 2003.

[3] K. Daskalakis and C.H. Papadimitriou. "The Complexity of Games on Highly Regular Graphs" 13th Annual Symposium on Algorithms (ESA) 2005.

[4] R.S. Datta. Algebraic Methods in Game Theory. PhD Dissertation, Department of Mathematics, U.C. Berkeley, 2003.

[5] A. Fabrikant, C.H. Papadimitriou and K. Talwar. "The Complexity of Pure Nash Equilibria", Proceedings of 36th STOC pp. 604-612, Chicago, Illinois, USA, Jun 2004. 
[6] G. Gottlob, G. Greco and F. Scarcello. "Pure Nash Equilibria: Hard and Easy Games", Proceedings of TARK IX (Theoretical Aspects of Rationality and Knowledge) pp. 215-230, Bloomington, Indiana, USA, Jun 2003.

[7] I. Gilboa and E. Zemel. "Nash and correlated equilibria: Some complexity considerations" Games and Economic Behavior, 1989.

[8] M. Kearns, M. Littman and S. Singh. "Graphical Models for Game Theory", Proceedings of UAI, 2001.

[9] R. Lipton and E. Markakis. "Nash Equilibria via Polynomial Equations" Proceedings of the 6th Latin American Symposium on Theoretical Informatics (LATIN'04), pp. 413-422, Buenos Aires, Argentina, 2004.

[10] M. Littman, M. Kearns and S. Singh. "An Efficient Exact Algorithm for Single Connected Graphical Games" Proceedings of 14th NIPS (Advances in Neural Information Processing Systems), pp. 817-823, 2002.

[11] J. Nash. "Noncooperative Games", Annals of Mathematics, 54, 289-295, 1951.

[12] J. von Neumann and O. Morgenstern. Theory of Games and Economic Behavior, Princeton University Press, 1944.

[13] C. H. Papadimitriou. "Computing Correlated Equilibria in Multiplayer Games" Proceedings of STOC, 2005.

[14] C. H. Papadimitriou. "On the Complexity of the Parity Argument and Other Inefficient Proofs of Existence," J. Comput. Syst. Sci. 48, 3, pp. 498-532, 1994.

[15] G.R. Schoenebeck and S.P. Vadhan. "The Computational Complexity of Nash Equilibria in Concisely Represented Games" Electronic Colloquiium on Computational Complexity, Report No. 52, 2005. 
Input: Normal form game $\mathcal{G}$ having $r$ players, $n$ strategies per player, given by utilities $\left\{u_{s}^{p}: p \in[r], s \in[n]\right\}$

Output: 4-player Normal form game $\mathcal{G}^{\prime}$.

1. Let $\mathcal{G G}$ be a graphical game constructed from $\mathcal{G}$ according to Figure 3.

2. Color the graph $(V \cup W, E)$ (where $E$ are directed edges of the affects-graph) of $\mathcal{G G}$. Let $c(w)=1$ for all $W$-vertices $w$. Initially, let $c(v)=2$ for all $V$-vertices $v$.

3. Construct graphical game $\mathcal{G} \mathcal{G}^{\prime}$ from $\mathcal{G G}$ as follows. While there exist $v_{1}, v_{2} \in V$, $w \in W,\left(v_{1}, w\right),\left(v_{2}, w\right) \in E$ with $c\left(v_{1}\right)=c\left(v_{2}\right):$

(a) Every $W$-vertex has at most 1 outgoing edge, so assume $\left(w, v_{1}\right) \notin E$.

(b) Add $v\left(v_{1}\right)$ to $V$, add $w\left(v_{1}\right)$ to $W$.

(c) Replace $\left(v_{1}, w\right)$ with $\left(v_{1}, w\left(v_{1}\right)\right),\left(w\left(v_{1}\right), v\left(v_{1}\right)\right),\left(v\left(v_{1}\right), w\right)$. Let $c\left(w\left(v_{1}\right)\right)=1$, choose $c\left(v\left(v_{1}\right)\right) \in\{2,3,4\} \neq c\left(v^{\prime}\right)$ for any $v^{\prime}$ with $\left(v^{\prime}, w\right) \in E$. Payoffs for $w\left(v_{1}\right)$ and $v\left(v_{1}\right)$ are chosen using Proposition 1 with $\alpha=1$ such that in any Nash equilibrium, $\mathbf{p}\left[v\left(v_{1}\right)\right]=\mathbf{p}\left[v_{1}\right]$.

4. For $i \in[4]$ let $V_{i}$ denote the set of vertices with color $i$. Assume all sets $V_{i}$ have the same cardinality $N$. (If necessary add idle isolated vertices.) If a vertex $v$ has fewer than 3 incoming edges, add extra incoming edges to $v$ so that $v$ has an incoming edge from each other color class.

5. Construct a 4-player game with $2 N$ strategies per player. Let $M=N^{2}$. For $j \in[N]$,

(a) If player 1 plays $2 j$ or $2 j+1$ and player 2 plays $2 j$ or $2 j+1$ then player 1 gets a payoff of $M$ and player 2 gets a payoff of $-M$.

(b) If player 3 plays $2 j$ or $2 j+1$ and player 4 plays $2 j$ or $2 j+1$ then player 3 gets a payoff of $M$ and player 4 gets a payoff of $-M$.

6. Let $V_{i}=\left\{v_{1}^{(i)}, \ldots, v_{N}^{(i)}\right\}$. For vertex $v_{j}^{(i)}$, let $v_{j^{\prime}}^{\left(i^{\prime}\right)}, v_{j^{\prime \prime}}^{\left(i^{\prime \prime}\right)}, v_{j^{\prime \prime \prime}}^{\left(i^{\prime \prime \prime}\right)}$ be the vertices that have edges going to $v_{j}^{(i)}$. The payoffs to $v_{j}^{(i)}$ depend on the actions of these 4 vertices. For $s$ a pure profile of these vertices, let $u_{s}\left(v_{j}^{(i)}\right)$ be the payoff to $v_{j}^{(i)}$ resulting from $s$. If $s$ is the binary numbers $\left(b, b^{\prime}, b^{\prime \prime}, b^{\prime \prime \prime}\right)$, where $b$ is the action chosen by $v_{j}^{(i)}$, and so on, give an additional payoff of $u_{s}\left(v_{j}^{(i)}\right)$ to player $c\left(v_{j}^{(i)}\right)$ if player $c\left(v_{j}^{(i)}\right)$ plays $j+b$, player $c\left(v_{j^{\prime}}^{\left(i^{\prime}\right)}\right)$ plays $j+b^{\prime}$, player $c\left(v_{j^{\prime \prime}}^{\left(i^{\prime \prime}\right)}\right)$ plays $j+b^{\prime \prime}$, player $c\left(v_{j^{\prime \prime \prime}}^{\left(i^{\prime \prime \prime}\right)}\right)$ plays $j+b^{\prime \prime \prime}$.

Figure 4: Reduction from normal form game $\mathcal{G}$ to 4 -player game $\mathcal{G}^{\prime}$ 


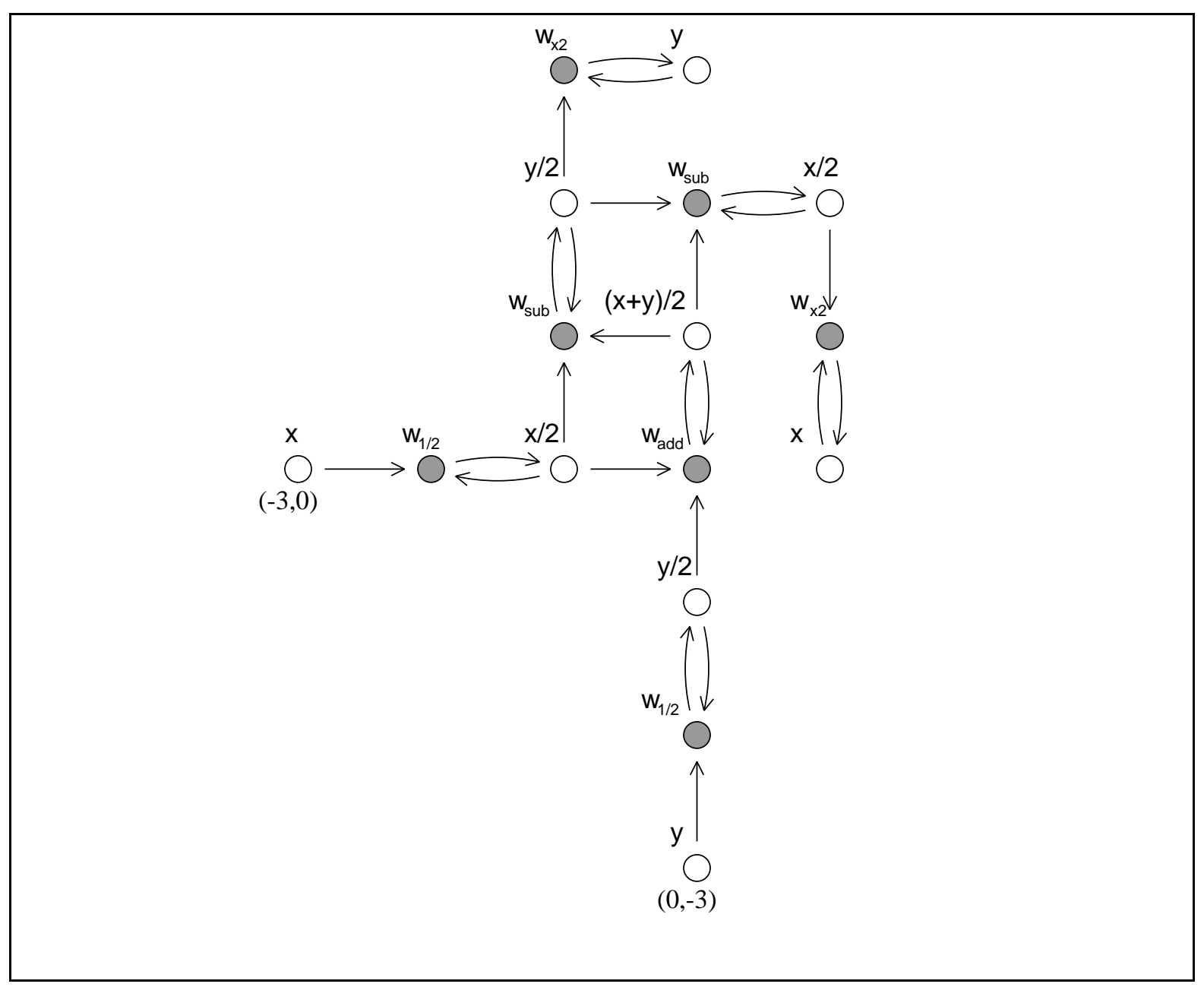

Figure 5: Diagram of Path Crossing in Grid

$\mathrm{ftp} / / / \mathrm{ftp}$ eccc.uni-trier.de/pub/eccc

ftpmail@ftp.eccc.uni-trier.de, subject 'help eccc' 